\title{
Thermalized ground state of artificial kagome spin ice building blocks
}

\author{
Unnar B. Arnalds, ${ }^{1}$ Alan Farhan, ${ }^{2}$ Rajesh V. Chopdekar, ${ }^{2}$ Vassilios Kapaklis, ${ }^{1}$ Ana Balan, ${ }^{2}$ \\ Evangelos Th. Papaioannou, ${ }^{1}$ Martina Ahlberg, ${ }^{1}$ Frithjof Nolting, ${ }^{2}$ Laura J. Heyderman, ${ }^{2}$ \\ and Björgvin Hjörvarsson ${ }^{1}$ \\ ${ }^{1}$ Department of Physics and Astronomy, Uppsala University, Box 516, SE-75120 Uppsala, Sweden \\ ${ }^{2}$ Paul Scherrer Institut, CH-5232 Villigen PSI, Switzerland
}

(Received 20 August 2012; accepted 27 August 2012; published online 11 September 2012)

\begin{abstract}
We present a direct magnetic imaging study on the thermal macrospin ordering of artificial kagome spin ice building blocks. Using photoemission electron microscopy, employing x-ray magnetic circular dichroism, we are able to resolve the single domain magnetic nature of the macrospins and determine the states of the combined building block structures. The nano-patterning and material selection allows thermally activated magnetization reversal for the macrospins to occur. The ordering of the macrospins is dominated by the ground state, consistent with a thermal ground state ordering. This work paves the way for the realization of extended artificial spin ice structures exhibiting experimentally accessible thermal behavior. (C) 2012 American Institute of Physics. [http://dx.doi.org/10.1063/1.4751844]
\end{abstract}

Artificial spin ice systems, ${ }^{1}$ composed of arrays of lithographically defined elongated ferromagnetic islands, have proven to be an interesting analog to bulk spin ice, ${ }^{2-4}$ which has a large set of nearly identical low-lying energy states with intricate thermodynamic properties. ${ }^{4}$ In artificial spin ice, it is possible to image directly the magnetic configurations using magnetically sensitive imaging techniques, such as magnetic force microscopy (MFM) and photoemission electron microscopy (PEEM). ${ }^{1,5}$ Using such techniques, the state of individual macrospins and vertices can be determined, which has resulted in the confirmation of the BernalFowler ice rules ${ }^{6}$ in artificial square spin ice ${ }^{1}$ and in the observation of emergent magnetic monopoles in artificial kagome spin ice. ${ }^{5}$

Studies on artificial spin ice have mostly focused on patterned structures composed of magnetic materials with a high Curie temperature such as permalloy $\left(\mathrm{Ni}_{81} \mathrm{Fe}_{19}\right)$ and cobalt. The high Curie temperature of these materials means that the spin ice systems are quasi-static at and below room temperature and, as a consequence, the manipulation of the magnetic ordering is limited to the use of external fields. ${ }^{1,5,7-9}$ Thermal behavior of artificial spin ice systems relies on the thermally activated reversal of the magnetization direction, where the energy barrier is defined by the shape anisotropy of the elements. Such reversal has been shown to be in accordance with the Néel-Brown model. ${ }^{10-13}$ The possibility for a thermally obtained ground state, as well as thermal excitations, therefore exists for artificial spin ice. The presence of long range ordering has already been observed in a square spin ice system in which the thermalization took place during the growth of the sample. ${ }^{14}$ Thermally driven order-disorder transitions of artificial spin ice have been studied theoretically ${ }^{15}$ and recently such a transition was reported experimentally in an artificial square spin ice. ${ }^{16}$

Here, we report on the observation of the thermally obtained ground state of dipole coupled nanomagnetic islands. We focus our study on hexagonal ring structures (see Fig. 1) composed of 6 macrospins, extending on to linked structures composed of two and three rings. These structures can be considered as the building blocks of artificial kagome spin ice. ${ }^{17,18}$ We investigate the magnetic ordering using PEEM, employing $\mathrm{x}$-ray magnetic circular dichroism (XMCD). This enables us to spatially resolve the magnetic states of the individual islands and the magnetic arrangement of each combined structure.

The islands are fabricated from $\delta$-doped $\mathrm{Pd}(\mathrm{Fe})$ films, in which the Curie temperature can be controlled by the Fe layer thickness. ${ }^{19,20}$ The Fe layer thickness was chosen to be 1.8 monolayers (ML), embedded in Pd layers as illustrated in Fig. 1. For these films, the remanent magnetization collapses at a temperature of $347 \mathrm{~K}$ while the saturation magnetization extends up to $374 \mathrm{~K}$. The films were patterned by electron beam lithography into one, two, and three ring kagome building block structures with a lattice parameter of $a=560 \mathrm{~nm}$ (Fig. 1). The magnetic moment of the individual islands can be determined from their area and the magnetization of the continuous film, determined by superconducting quantum interference device (SQUID) magnetometer measurements. For the islands described here, of length $470 \mathrm{~nm}$

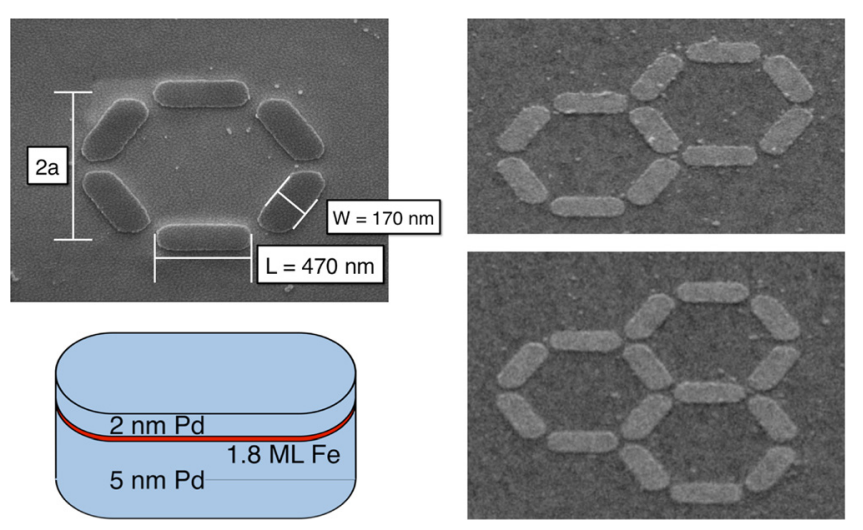

FIG. 1. Scanning electron microscope images (recorded at a tilt angle of $45^{\circ}$ ) showing the one, two, and three ring structures. The lateral dimensions of the individual islands are $W=170 \mathrm{~nm}$ and $L=470 \mathrm{~nm}$ and the lattice parameter of the arrangement is $a=560 \mathrm{~nm}$. The structures are spaced $3 \mu \mathrm{m}$ apart to minimize dipolar coupling. A schematic illustration of the inner structure of the islands is also included. 
(a)
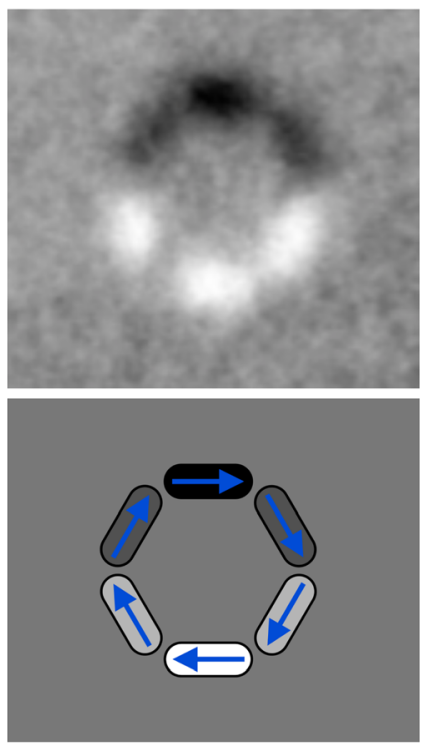

(b)

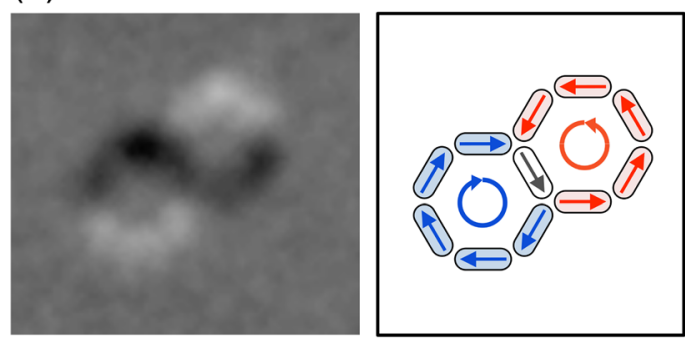

(c)

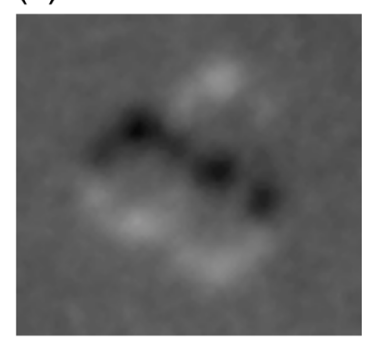

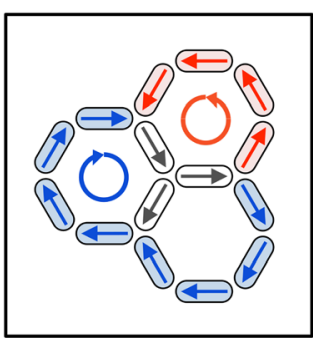

FIG. 2. XMCD images of the magnetic state of the ring structures recorded at $\sim 120 \mathrm{~K}$. (a) The clockwise vortex ground state ordering of a one ring structure. The schematic below illustrates the determination of the magnetic state of each of the islands from the magnetic contrast. XMCD images showing the lowest energy levels of (b) a two ring structure and (c) a three ring structure. and width $170 \mathrm{~nm}$, the resulting $0 \mathrm{~K}$ moment is $m_{0}=$ $7.2 \times 10^{-17} \mathrm{Am}^{2}\left(7.8 \times 10^{6} \mu_{\mathrm{B}}\right)$.

The dipole energy of each state of the combined structures, $E_{n}$, can be calculated by summing up the dipole interactions between macrospins for all possible configurations. For the one ring structure, composed of 6 macrospins, the number of possible configurations is $2^{6}$. Due to the symmetry of the ring structure, the possible states can be classified into 8 different energy levels $E_{l}$, each with a degeneracy $d_{l}{ }^{17}$ The dipolar energy is minimized when neighboring macrospins are aligned head to tail. This corresponds to a lowest energy vortex state, of twofold degeneracy, in which the moments all point in a circular arrangement of either a clockwise or counterclockwise direction.

The distribution of the structures into the different magnetic states was determined by PEEM imaging at the SIM beamline $^{21,22}$ of the Swiss Light Source, employing XMCD

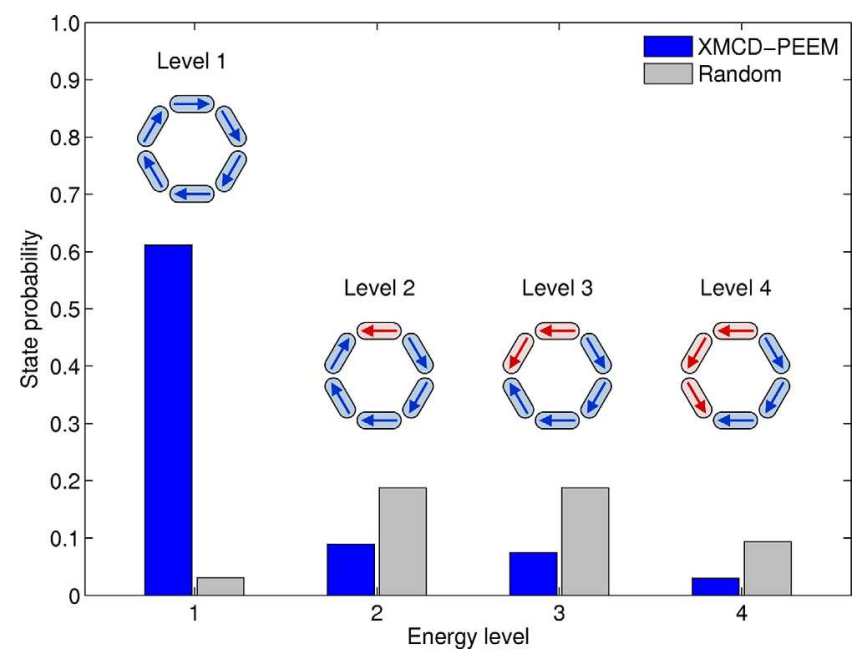

FIG. 3. The population of the four lowest energy levels determined from $\mathrm{XMCD}$ images. The ground state displays a higher probability compared to a random distribution, whereas higher energy levels display a lower probability. A total of 67 ring arrangements were imaged from which the state of a total of 54 ring structures could be identified. at the Fe $L_{3}$ edge. ${ }^{23}$ No magnetic field was applied prior to imaging. Imaging at room temperature did not reveal any magnetic contrast, although the magnetic moment has only been reduced to $\sim 40 \%$ of its $0 \mathrm{~K}$ value. Upon cooling from room temperature to cryogenic temperatures $(\sim 120 \mathrm{~K})$ using a continuous flow of liquid nitrogen, the islands displayed a distinct magnetic contrast. Figure 2 shows representative XMCD images of the one, two, and three ring structures. It should be noted that the magnetic contrast from these ultrathin Fe films sitting below a Pd layer is significantly smaller than that observed in islands manufactured from several nanometer thick magnetic films. ${ }^{17}$

An overview of the observed population of each of the possible levels of the one ring structures is shown in Fig. 3. The probabilities are determined from XMCD images as illustrated in Fig. 2. As seen in the graph, the vortex ground

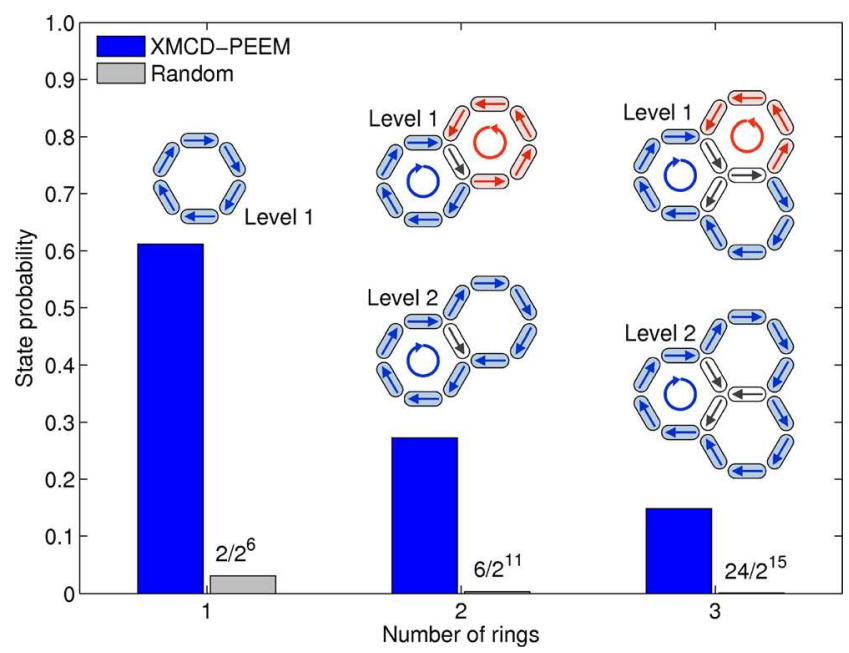

FIG. 4. The population of the ground state of the one ring structure and of the two lowest energy levels for the two and three ring structures (depicted in the graph). In all cases, the measured probabilities are higher than those expected from a random distribution. A total of 55 and 54 structures were imaged for the two and three ring structures, respectively, from which the state of a total of 39 and 31 structures could be identified. 

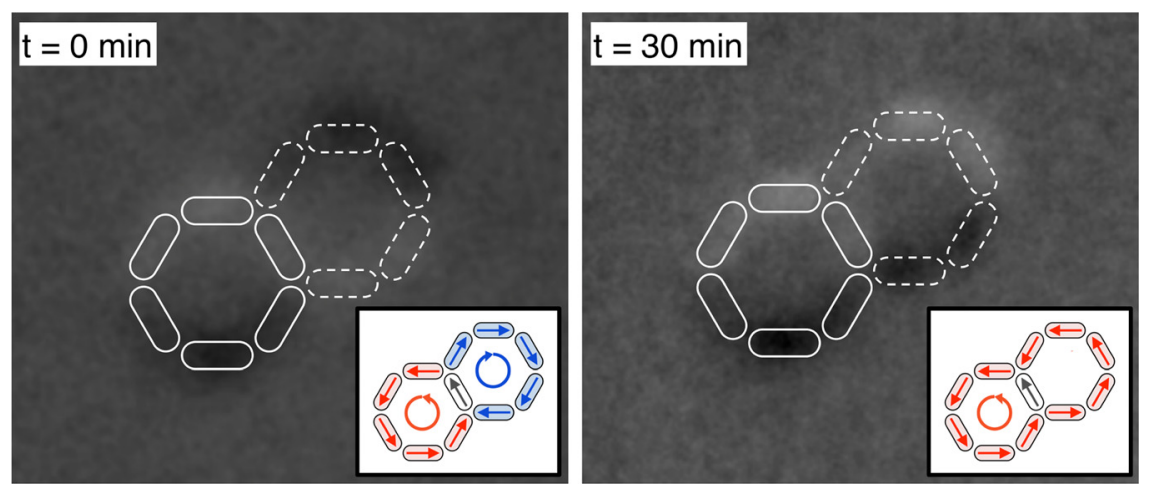

FIG. 5. XMCD images of a two ring structure showing the macrospins changing direction between the two energy minima. Over a period of $30 \mathrm{~min}$, the state of the structure evolved from the lowest energy ground state to the second lowest energy level. Borders are drawn on to the images to highlight the location and arrangement of the islands. The macrospins which display a reversal of the magnetization direction are marked with a dashed white border.

state is clearly favored. Full randomization would result in the probability of observed states given by the degeneracy of each possible state, divided by the total number of possible arrangements, $2^{6}$. However, the high energy states are less probable than would be expected for a random distribution and the four highest energy levels were never observed. Finally, no specific preference for the clockwise or counterclockwise direction of the ground state was observed. The results therefore clearly demonstrate the preferred population of low energy states, consistent with a thermal ordering induced by the dipolar interactions between the islands.

As the number of elements, $L$, is increased, the number of available configurations, $2^{L}$, grows dramatically. For the two and three ring structures composed of 11 and 15 elements, respectively, a total of $2^{11}=2048$ and $2^{15}=32768$ different configurations are obtained. A detailed analysis of the distribution into all the possible arrangements therefore requires a substantial amount of states to be observed in order to obtain statistically significant results. We therefore restrict our analysis to the population of the two lowest energy levels. For the two ring structure, the lowest energy level is composed of two circular arrangements of macrospins (vortices) of opposite chirality with a 2 fold degeneracy. The second lowest energy level, of 4 fold degeneracy, is referred to as an external flux closure state ${ }^{17}$ and comprises one full vortex and one in which the magnetization direction of one macrospin has been reversed. The two lowest energy levels of the three ring structure can be described in a similar manner with the ground state composed of two vortices with the third ring not quite forming a vortex and the second lowest energy level as an external flux closure state, both of 12 fold degeneracy (see Figs. 2(b) and 2(c) and schematics in Fig. 4). ${ }^{17}$

The combined population of the two lowest energy states of the two and three ring structures, determined from $\mathrm{XMCD}$ images of arrays of the two and three ring structures, is shown in Fig. 4. The results reveal a significantly higher population of the lowest energy states than would be expected given a random distribution $\left(6 / 2^{11}\right.$ and $24 / 2^{15}$, respectively). The probability of obtaining the lowest energy states diminishes with increasing number of rings.

To demonstrate thermally activated behavior in artificial spin ice systems, the macrospins must exhibit dynamics on the time scale of the experiment, which would yield spontaneous transitions between different states as measured by changes in PEEM-XMCD contrast of the $\mathrm{Pd}(\mathrm{Fe})$ structures as a function of time. Transitions of this kind were observed in the experiment by monitoring the magnetic state over an extended time, verifying the thermal behavior of the building block structures at $\sim 120 \mathrm{~K}$. This is illustrated in Fig. 5 which shows a transition of the arrangement of the macrospins in a two ring structure, from the lowest energy ground state, to the second lowest energy state.

In conclusion, we have demonstrated thermal ordering in dipole coupled macrospin systems using thermal cycling. At elevated temperatures, the macrospins fluctuate, resulting in the absence of magnetic contrast at $300 \mathrm{~K}$. At low temperatures, the macrospins fluctuate with a reduced rate, allowing for the direct imaging determination of their arrangement and the observation of thermal transitions between different states. Given these results, thermal behavior can be obtained for extended arrays of dipole coupled macrospins in an artificial spin ice geometry. This opens the door for experimentally determining thermodynamic properties such as entropy and ground state ordering, and for the observation of thermal behavior of charge defects that can be investigated in the context of emergent magnetic monopoles. $5,24,25$

The authors acknowledge the support of the Swedish Research Council (VR), the Knut and Alice Wallenberg Foundation (KAW), the Swiss National Science Foundation (SNF), and the Icelandic Research Fund for Graduate Students. Part of this work was performed at the Swiss Light Source, Paul Scherrer Institut, Villigen, Switzerland. We wish to thank Anja Weber for development of the patterning processes and sample manufacture. We are also grateful to Vitaliy Guzenko for support with electron beam lithography.

${ }^{1}$ R. F. Wang, C. Nisoli, R. S. Freitas, J. Li, W. McConville, B. J. Cooley, M. S. Lund, N. Samarth, C. Leighton, V. H. Crespi, and P. Schiffer, Nature 439, 303 (2006).

${ }^{2}$ M. J. Harris, S. T. Bramwell, D. F. McMorrow, T. Zeiske, and K. W. Godfrey, Phys. Rev. Lett. 79, 2554 (1997).

${ }^{3}$ A. P. Ramirez, A. Hayashi, R. J. Cava, R. Siddharthan, and B. S. Shastry, Nature 399, 333 (1999).

${ }^{4}$ S. T. Bramwell and M. J. P. Gingras, Science 294, 1495 (2001).

${ }^{5}$ E. Mengotti, L. J. Heyderman, A. Fraile Rodríguez, F. Nolting, R. V. Hügli, and H. B. Braun, Nat. Phys. 7, 68 (2011).

${ }^{6}$ J. D. Bernal and R. H. Fowler, J. Chem. Phys. 1, 515 (1933).

${ }^{7}$ C. Nisoli, R. Wang, J. Li, W. F. McConville, P. E. Lammert, P. Schiffer, and V. H. Crespi, Phys. Rev. Lett. 98, 217203 (2007).

${ }^{8}$ X. Ke, J. Li, C. Nisoli, P. E. Lammert, W. McConville, R. F. Wang, V. H. Crespi, and P. Schiffer, Phys. Rev. Lett. 101, 037205 (2008).

${ }^{9}$ Z. Budrikis, P. Politi, and R. L. Stamps, Phys. Rev. Lett. 105, 017201 (2010).

${ }^{10}$ L. Néel, Ann. Geophys. 5, 99 (1949).

${ }^{11}$ W. F. Brown, Phys. Rev. 130, 1677 (1963).

${ }^{12}$ W. Wernsdorfer, E. B. Orozco, K. Hasselbach, A. Benoit, B. Barbara, N. Demoncy, A. Loiseau, H. Pascard, and D. Mailly, Phys. Rev. Lett. 78, 1791 (1997). 
${ }^{13}$ R. H. Koch, G. Grinstein, G. A. Keefe, Y. Lu, P. L. Trouilloud, W. J. Gallagher, and S. S. P. Parkin, Phys. Rev. Lett. 84, 5419 (2000).

${ }^{14}$ J. P. Morgan, A. Stein, S. Langridge, and C. H. Marrows, Nat. Phys. 7, 75 (2011).

${ }^{15}$ R. C. Silva, F. S. Nascimento, L. A. S. Mól, W. A. Moura-Melo, and A. R. Pereira, New J. Phys. 14, 015008 (2012).

${ }^{16}$ V. Kapaklis, U. B. Arnalds, A. Harman-Clarke, E. Th. Papaioannou, M. Karimipour, P. Korelis, A. Taroni, P. C. W. Holdsworth, S. T. Bramwell, and B. Hjörvarsson, New J. Phys. 14, 035009 (2012).

${ }^{17}$ E. Mengotti, L. J. Heyderman, A. Fraile Rodríguez, A. Bisig, L. Le Guyader, F. Nolting, and H. B. Braun, Phys. Rev. B 78, 144402 (2008).

${ }^{18}$ L. J. Heyderman, T. Jung, E. Mengotti, A. Bisig, A. Fraile Rodríguez, F. Nolting, H. B. Braun, and T. Schrefl, U.S. patent 8,085,578 B2 (2011).
${ }^{19}$ M. Pärnaste, M. Marcellini, E. Holmström, N. Bock, J. Fransson, O. Eriksson, and B. Hjörvarsson, J. Phys. Condens. Matter 19, 246213 (2007).

${ }^{20}$ E. Th. Papaioannou, V. Kapaklis, A. Taroni, M. Marcellini, and B. Hjörvarsson, J. Phys. Condens. Matter 22, 236004 (2010).

${ }^{21}$ L. Le Guyader, A. Kleibert, A. Fraile Rodríguez, S. E. Moussaoui, A. Balan, M. Buzzi, J. Raabe, and F. Nolting, "Studying nanomagnets and magnetic heterostructures with X-ray PEEM at the Swiss Light Source," J. Electron Spectrosc. Relat. Phenom. (in press), doi:10.1016/j.elspec.2012.03.001.

${ }^{22}$ U. Flechsig, F. Nolting, A. Fraile Rodríguez, J. Krempasky, C. Quitmann, T. Schmidt, S. Spielmann, and D. Zimoch, AIP Conf. Proc. 1234, 319 (2010).

${ }^{23}$ J. Stöhr, Y. Wu, B. D. Hermsmeier, M. G. Samant, G. R. Harp, S. Koranda, D. Dunham, and B. P. Tonner, Science 259, 658 (1993).

${ }^{24}$ C. Castelnovo, R. Moessner, and S. L. Sondhi, Nature 451, 42 (2008).

${ }^{25}$ S. R. Giblin, S. T. Bramwell, P. C. W. Holdsworth, D. Prabhakaran, and I. Terry, Nat. Phys. 7, 252 (2011). 